\title{
Value of Preoperative Analysis Using Three-Dimensional Imaging of Leg Deformity after Injury to the Distal Femoral Epiphysis
}

Kenji Tobita ${ }^{1 *}$, Hiroshi Okazaki ${ }^{2}$, Yoshinao Koike ${ }^{2}$, Yoshiharu Simozono ${ }^{2}$, Masahiko Bessho ${ }^{3}$, Sakae Tanaka ${ }^{4}$ and Isao Ohnishi ${ }^{3}$

${ }^{1}$ Nishi-Shinjuku Bone Clinic, Tokyo, Japan

${ }^{2}$ Kanto Rosai Hospital, Kanagawa, Japan

International University of Health and Welfare, Tochigi, Japan

${ }^{4}$ Department of Orthopaedic Surgery, Faculty of Medicine University of Tokyo, Japan

\begin{abstract}
Background: Assessment of lower extremity alignment is important. The malalignment test is generally assessed two-dimensionally on plain radiographs. In patients with complex deformities, the key points are set inaccurately on Roentgenogram images, leading to problems with both accuracy and reproducibility. Computed tomography (CT) is the current gold standard for evaluating femoral torsion measurements. However, CT images vary depending on slice orientation during scanning, images of flexion deformities are affected by the orientation of the CT slice, making accurate evaluation impossible should a flexion deformity be present. For this reason, three-dimensional preoperative analysis is required. We report a case in which three-dimensional preoperative analysis was used for a patient with leg deformity after injury to the distal femoral epiphysis, with good results.
\end{abstract}

Methods: CT scanning was used to establish a preoperative plan and produced a surface morphology model from CT axial data. Free software was then used to create mirror images of the affected and unaffected sides from these data. The mirror image of the unaffected side was then positioned in three-dimensional space, and vectors were set. The fragment proximal to the osteotomy plane was defined as the reference fragment (RF) and the distal fragment as the corresponding fragment (CF), with the functional axis of the unaffected side forming the helical axis $(\mathrm{HA})$. The amount of deformity correction of the CF required was then calculated automatically in terms of the two parameters of rotation and translation in three dimensions by shifting the CF along the HA. A Taylor spatial frame (TSF) was used to perform gradual correction surgery.

Results: The rotational deformity was corrected, a 20-mm medial shift and 12-mm posterior shift persisted. Correction was carried out a second time to correct this deformity.

Conclusion: Three-dimensional analysis of long-bone may prove useful for the correction of long bones deformities in the legs when complex deformity is present.

keywords: Distal femoral epiphysis; Limb deformities; Computed tomography; Digital imaging and communication in medicine

\section{Introduction}

Limb deformities have a variety of causes, including malunion after fracture, congenital deformity, and osteoarthritis. Such deformities are not only problematic from the viewpoint of external appearance, but also impair movement and stability of adjacent joints, and may cause pain and progression of arthritis [1]. Accurate deformity correction is required to improve such disabilities. The malalignment test proposed by Paley et al. [2,3] is currently used to preoperatively evaluate deformity for orthomorphic surgery of the legs. In this method, roentgenograms are taken and a number of key points are selected on the images, and the angles formed by the line segments joining the center of the femoral head to the greater trochanter and that picking up the distal point of the condyle are defined and calculated proximally as the Lateral Proximal Femoral Angle (LPFA) and distally as the Lateral Distal Femoral Angle (LDFA). Normal values have been determined from evaluations of large numbers of healthy bones, and in the correction of deformity these parameters are calculated for the deformed bones and orthomorphic surgery is planned so as to restore normal values. Paley's method of setting the key points, however, may result in their being set inaccurately on Roentgenogram images of patients with complex deformities [4]. Evaluate rotational deformities is also not possible on Roentgenograms. Two Computed Tomography (CT) cross-sectional slice images are therefore used for analysis $[5,6]$. The rotation angle is defined and evaluated as the angle formed by the line segment from the center of the femoral head that passes through the center of the neck and that joining the posterior-most points of the medial and lateral femoral condyles. Because CT images vary depending on slice orientation during scanning, however, images of flexion deformities are affected by the orientation of the CT slice, making accurate evaluation impossible should a flexion deformity be present, although this is not a problem in the absence of such deformities (Figure 1).

In the current method of analysis of rotational deformity using two-dimensional CT, the images acquired during CT thus vary as a result of leg position and flexion deformity. In patients with complex deformities, the key points are set inaccurately on Roentgenogram images, leading to problems with both accuracy and reproducibility. Three-dimensional preoperative analysis is therefore required.

When carrying out deformity correction in three dimensions,

*Corresponding author: Kenji Tobita, Nishi-Shinjuku Bone Clinic, Tokyo, Japan, Tel: 0120-962-992; E-mail: tobitak.ort@gmail.com

Received January 26, 2015; Accepted April 22, 2015; Published April 26, 2015

Citation: Tobita K, Okazaki H, Koike Y, Simozono Y, Bessho M, et al. (2015) Value of Preoperative Analysis Using Three-Dimensional Imaging of Leg Deformity after Injury to the Distal Femoral Epiphysis. J Trauma Treat 4: 246. doi:10.4172/21671222. 1000246

Copyright: ( 2015 Tobita K, et al. This is an open-access article distributed under the terms of the Creative Commons Attribution License, which permits unrestricted use, distribution, and reproduction in any medium, provided the original author and source are credited. 
Citation: Tobita K, Okazaki H, Koike Y, Simozono Y, Bessho M, et al. (2015) Value of Preoperative Analysis Using Three-Dimensional Imaging of Leg Deformity after Injury to the Distal Femoral Epiphysis. J Trauma Treat 4: 246. doi:10.4172/2167-1222.1000246

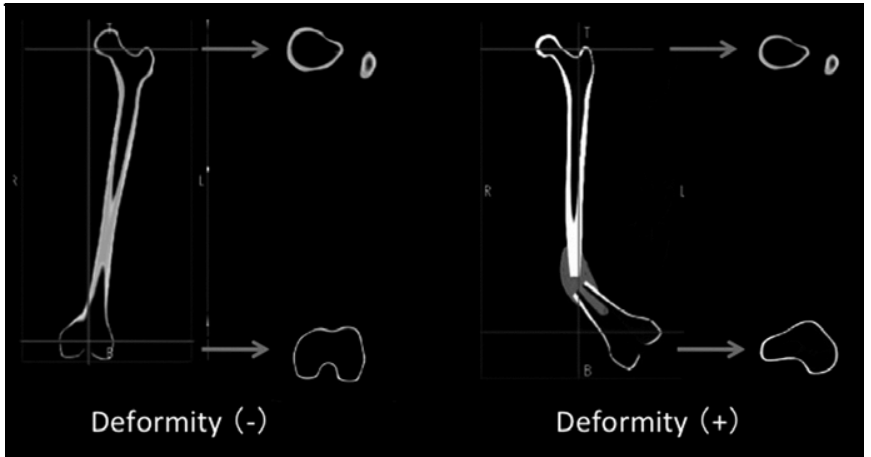

Figure 1: CT images vary depending on slice orientation during scanning, however, images of flexion deformities are affected by the orientation of the CT slice, making accurate evaluation impossible should a flexion deformity be present, although this is not a problem in the absence of such deformities.

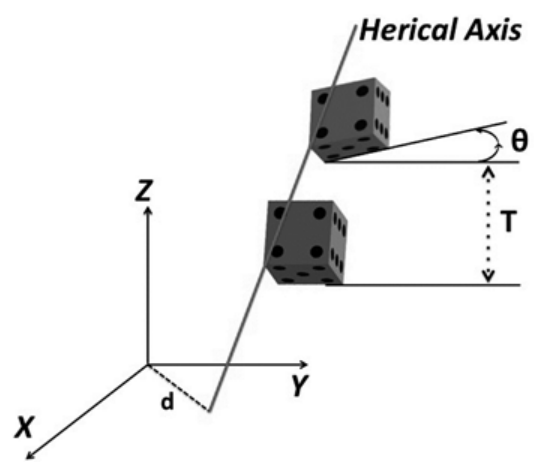

Figure 2: Rotation and translation can be performed simultaneously along the helical axis. All objects are present in the three-dimensional space, and the position of a body can be freely shifted by moving it along this axis.

the concept of the helical axis is used (Figure 2) [7,8]. Use of this concept enables quantitative evaluation of the structural elements of the deformity in three dimensions in terms of the two parameters of rotation and translation. We report herein a case in which threedimensional preoperative analysis was used for a patient with leg deformity after injury to the distal femoral epiphysis, with good results.

\section{Material and Methods}

The patient was a girl who was first examined in our hospital at 16 years old after having been diagnosed with injury to the distal femoral epiphysis at 12 years old.

\section{Findings on initial examination}

Although the patient was capable of walking without an orthosis, limited mobility associated with pain on the posterolateral side of the knee was evident after walking for extended periods. Range of motion of the knee was unrestricted, with right and left flexion of $150^{\circ}$ and extension of $0^{\circ}$, and there was no obvious manual laxity of the medial and lateral collateral ligaments or the anterior cruciate ligament. In imaging findings on initial examination (Figure 3a-3c), knee Roentgenograms showed valgus deformity at the left distal femur, with a 24-mm discrepancy in leg length evident on Roentgenograms of the full length of both legs.

\section{Preoperative plan using CT}

CT scanning (Aquilion Super 4; Toshiba Medical Systems, Tokyo,
Japan) was used to establish a preoperative plan. The full length of both femurs was scanned, with imaging conditions of $120 \mathrm{kV}, 150 \mathrm{~mA}$ $\mathrm{s}$ and $0.185 \mathrm{~mm}$ pixels. After scanning, CT image data were saved in digital imaging and communication in medicine (DICOM) format, and software (Zed View; LEXI Co., Ltd. Tokyo, Japan) was used to produce a surface morphology model from axial data, which was saved as Standard Triangulated Language (STL) data. Free software (Studio Basic; netfabb, Lupburg, Germany) was then used to create mirror image of the unaffected side from these data (Figure 4a). Two images were superimposed (Figure $4 \mathrm{~b}$ ), enabling the deformation plane, i.e., the plane of rotation of angulation (PORA), to be easily isolated (Figure 4c). This plane was used as the location for osteotomy.

The mirror image of the unaffected side was then positioned in three-dimensional space, and vectors were set with the functional axis of the unaffected femur as the Z-axis, the mediolateral orientation of the bone as the $\mathrm{X}$-axis, and the $\mathrm{Y}$-axis perpendicular to the $\mathrm{XZ}$ plane. The origin was set at the center of the PORA (Figure $4 \mathrm{~d}$ ). The fragment proximal to the PORA was defined as the Reference Fragment (RF) and the distal fragment as the Corresponding Fragment (CF), with the functional axis of the unaffected side forming the Helical Axis (HA). The amount of deformity correction of the CF required was then

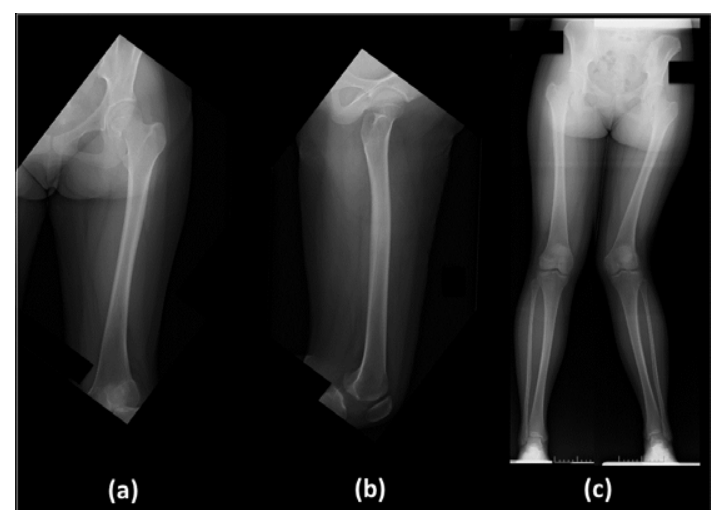

Figure 3: a-c) Preoperative anteroposterior (a), lateral (b) and anteroposterior view standing radiographs (c) show valgus and rotational deformities of the distal end of the femur, along with leg length discrepancy.

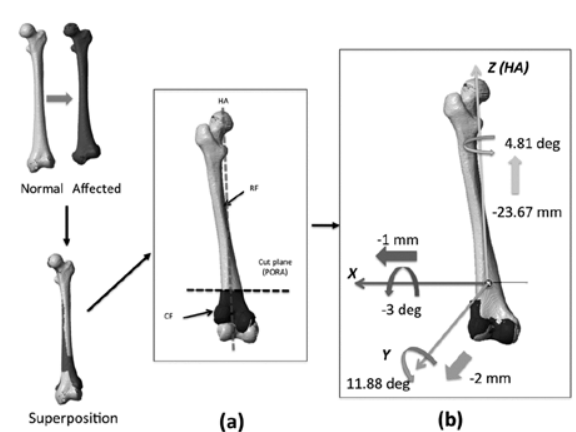

Figure 4: The plane in which the proximal part of the intact femur and distal part of the affected femur intersect is called the plane of rotation of angulation (PORA).

a) The center of gravity for $X Y Z$ coordinate was defined as the center of the PORA. The $Z$ axis was defined to coincide with the long axis of the femur. The $Y$ axis was defined as the mediolateral $(M L)$ direction of the femur. The $X$ axis was defined as perpendicular to the $Y Z$ plane and was directed anteroposterior $(A P)$ on the femur.

b) Reference fragment (RF), corresponding fragment (CF) and helical axis (HA) are shown. Displacement of CF can be reduced into a rotation about and a slide along this axis 
Citation: Tobita K, Okazaki H, Koike Y, Simozono Y, Bessho M, et al. (2015) Value of Preoperative Analysis Using Three-Dimensional Imaging of Leg Deformity after Injury to the Distal Femoral Epiphysis. J Trauma Treat 4: 246. doi:10.4172/2167-1222.1000246

calculated automatically in terms of the two parameters of rotation and translation in three dimensions by shifting the CF along the HA.

\section{Surgery}

The Taylor Spatial Frame (TSF) (Smith \& Nephew, Inc., Memphis, TN, USA) was used to perform gradual correction surgery. The leg was rotated so that the patella was facing forward. Using fluoroscopic imaging, two 6-mm half pins (Smith and Nephew, Memphis, Tennessee) were advanced across the proximal femur from lateral to medial in the coronal plane, perpendicular to the anatomical axis of proximal femur and the proximal full ring was centred on the femur. The ring was held perpendicular to the anatomical axis of the femur in the sagittal and plane.

The distal ring was then placed with the appropriate strut length taken into account. First 1.8-mm smooth wire was inserted from lateral epicondyle to medial epicondyle so as to parallel to the knee joint surface. The distal ring was centred on the leg and fastened to the wire. The wire was then tensioned. Second wire was passed from the posterolateral to anteromedial. A third wire was placed between these two wires. Six struts were attached to the proximal and distal rings, and the strut settings were noted and locked. An oblique osteotomy was carried out at the PORA identified during preoperative planning (Figure $5 \mathrm{a}$ and $5 \mathrm{~b}$ ).

Postoperative CT scanning was used as the second measurement technique. CT image data were saved in DICOM format and was used to produce a STL.

The antero-posterior (Y-axis) and medio-lateral (X-axis) frame offset was calculated using the distance of the line from the midpoint to the point of origin on the axial view of the 3D STL model image. After a waiting period of 1 week after surgery, correction was started using the TSF deformity correction program.

\section{Results}

Figure 4 shows the preoperative deformity measured from CT images. Deformity correction was carried out on the basis of these parameters, but although the rotational deformity was corrected, a 20$\mathrm{mm}$ medial shift and $12-\mathrm{mm}$ posterior shift persisted (Figure $6 \mathrm{a}$ and $6 \mathrm{~b})$. Correction was carried out a second time to correct this deformity (Figure 7). The first correction period was 31 days, and the second was 10 days (total, 41 days). The external fixator was left in place to allow the bone to mature for a further 163 days before removal (Figure 8). Although mild extension contracture (5 degree) of the knee remained, Knee Society Score was 71 and Function Score was 100.

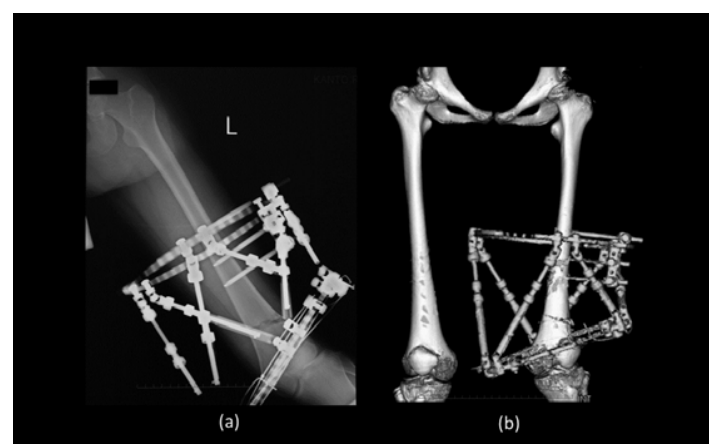

Figure 5: a, b) Postoperative anteroposterior radiograph (a) and threedimensional CT.

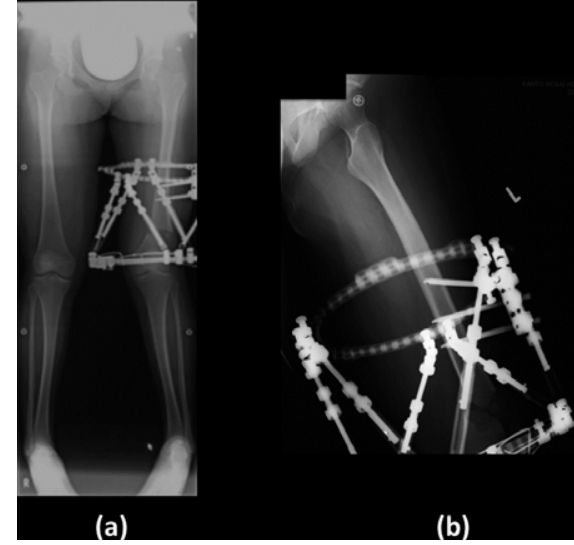

Figure 6: a, b) Anteroposterior (a) and lateral (b) radiographs after first deformity correction. The rotational deformity has been corrected. However, medial $(20 \mathrm{~mm})$ and posterior $(12 \mathrm{~mm})$ displacements remain.

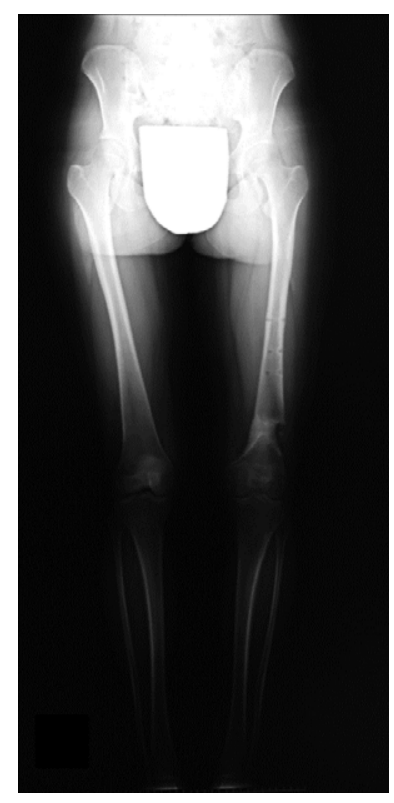

Figure 7: Appropriate correction was obtained, as evident on anteroposterior standing radiograph made after removal of the external fixator.

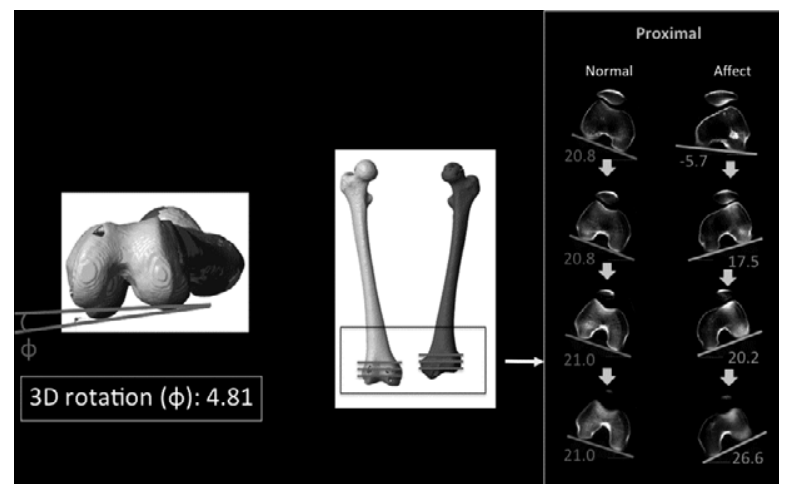

Figure 8: Evaluation of the rotation angle on three-dimensional image showed that the unaffected and affected legs differed by an angle of rotation of $4.81^{\circ}$, but in two-dimensional analysis on axial CT there was wide variation in this angle. 
Citation: Tobita K, Okazaki H, Koike Y, Simozono Y, Bessho M, et al. (2015) Value of Preoperative Analysis Using Three-Dimensional Imaging of Leg Deformity after Injury to the Distal Femoral Epiphysis. J Trauma Treat 4: 246. doi:10.4172/2167-1222.1000246

\begin{tabular}{|c|c|c|c|c|c|}
\hline Evaluation & $\begin{array}{c}\text { Valgus } \\
\text { (degree) }\end{array}$ & $\begin{array}{c}\text { LLD } \\
(\mathbf{m m})\end{array}$ & $\begin{array}{c}\text { Medial } \\
\text { Shift } \mathbf{( m m})\end{array}$ & $\begin{array}{c}\text { Anterior Convex } \\
\text { (degree) }\end{array}$ & $\begin{array}{c}\text { Anterior Shift } \\
(\mathbf{m m})\end{array}$ \\
\hline $\begin{array}{c}\text { Paley's } \\
\text { method }\end{array}$ & 20.3 & 24 & 77 & N/A & N/A \\
\hline $\begin{array}{c}\text { Current } \\
\text { method }\end{array}$ & 11.8 & 23.67 & 1 & 3 & 2 \\
\hline $\begin{array}{c}\Delta \\
\text { LLD: Leg Length Discrepancy. }\end{array}$ & 8.42 & 0.33 & 76 & N/A & N/A \\
\hline
\end{tabular}

Table 1: Comparison results of deformity angle between Paley's and current method.

\section{Discussion}

We used three-dimensional preoperative analysis of a deformity of the femoral condyle to perform deformity correction using a Taylor spatial frame. Comparison of analyses of the deformity using preoperative Roentgenograms and using the three-dimensional technique (Table 1) showed that the valgus deformation was estimated at $20.3^{\circ}$ on two-dimensional Roentgenograms, but $11.88^{\circ}$ when the three-dimensional technique was used, with three-dimensional valgus overestimated on frontal Roentgenogram images. There was no difference in the discrepancy in leg length calculated by the two techniques, but three-dimensional analysis showed only a very small mechanical axis deviation (MAD) of $1 \mathrm{~mm}$, compared to $77 \mathrm{~mm}$ on two-dimensional images. Because valgus was overestimated on these two-dimensional images, MAD was also overestimated. On lateral images, the medial and lateral condyles were displaced because of the valgus and rotational deformity, and analysis could not be performed using Roentgenogram images. Evaluation of the rotation angle on three-dimensional image showed that the unaffected and affected legs differed by an angle of rotation of $4.81^{\circ}$, but in two-dimensional analysis on axial CT there was wide variation in this angle, which had a standard deviation of $14^{\circ}$ on the affected side (Figure 8). The reason of this was that the distal valgus deformity of the femur affected the shape of the acquired images, meaning that the angle of rotation could not be accurately evaluated.

Two programs of correction were required in the present case, with the cause of persistent deformity after initial correction regarded as increased soft tissue tension during correction and errors in relative positions of the rings and femur.In terms of the use of threedimensional images for deformity correction of long bones, Murase et al. [9] previously used this technique to correct arm deformities. They performed preoperative three-dimensional cutting of hydroxyapatite block based on a simulation using three-dimensional computer-aided design, and used these customized implants in corrective osteotomy. In the present case, we used Murase's method as a reference when carrying out three-dimensional quantitative analysis of a leg deformity, and performed gradual correction of this deformity in three-dimensional space. The use of Roentgenograms for leg deformity correction of complex deformities is cumbersome, and frequent re-correction is required. Although one reprogramming was required using our method, three-dimensional analysis of long-bone may prove useful for the correction of long bones deformities in the legs when complex deformity is present.

\section{References}

1. Tetsworth K, Paley D (1994) Malalignment and degenerative arthropathy Orthop Clin North Am 25: 367-377.

2. Paley D, Herzenberg JE, Tetsworth K, McKie J, Bhave A (1994) Deformity planning for frontal and sagittal plane corrective osteotomies. Orthop Clin North Am 25: 425-465.

3. Paley D, Pfeil J (2000) Principles of deformity correction around the knee. Orthopade 29:18-38.

4. Ariumi A, Sato T, Kobayashi K, Koga Y, Omori G et al. (2010) Three-dimensional lower extremity alignment in the weight-bearing standing position in healthy elderly subjects. J Orthop Sci 15: 64-70.

5. Tomczak RJ, Guenther KP, Rieber A, Mergo P, Ros PR, et al. (1997) MR imaging measurement of the femoral antetorsional angle as a new technique: comparison with CT in children and adults. AJR Am J Roentgenol 168:791-794.

6. Waidelich HA, Strecker W, Schneider E (1992) Computed tomographic torsionangle and length measurement of the lower extremity. The methods, normal values and radiation load. Rofo 157: 245-251.

7. Kinzel GL, Hall AS, Jr., Hillberry BM (1972) Measurement of the total motion between two body segments. I. Analytical development. J Biomech. 5: 93-105.

8. Kinzel GL, Hillberry BM, Hall AS Jr., Van Sickle DC, Harvey WM (1972) Measurement of the total motion between two body segments. II. Description of application. J Biomech 5: 283-293.

9. Murase T, Oka K, Moritomo H, Goto A, Yoshikawa H, et al. (2008) Threedimensional corrective osteotomy of malunited fractures of the upper extremity with use of a computer simulation system. J Bone Joint Surg Am 90: 2375-2389. 